\title{
Four facets of rigor
}

\section{Mark B. Houston ${ }^{1}$}

Published online: 1 June 2019

(C) Academy of Marketing Science 2019

I'm honored to be joining Journal of the Academy of Marketing Science as co-editor to Editor-in-Chief John Hulland. My commitment to JAMS is certainly due to the journal's long-running role in our discipline. However, it is also a bit personal as my first professional publication was in JAMS (Houston and Walker 1996), and, throughout my career, JAMS has been a regular target for my work. After many years on the ERB and several years as an Area Editor, it is my goal as co-editor to help maintain the positive trajectory of JAMS as it continues to strengthen in reputation and recognition, being considered a top-tier outlet at a growing number of great schools and in journal rankings.

Why has JAMS continued to rise in esteem? In addition to a line of dedicated editors who have done their jobs with a strong vision for the journal, I believe that a big factor is JAMS' ongoing commitment to publishing research that has substantive importance and appropriate rigor. Interestingly, our field's rising standards regarding rigor have been a major topic among scholars; all recognize the importance of rigor, but some express concern that methodological rigor has become, in some cases, more important than the underlying research question (cf. Lehmann et al. 2011; Moorman et al. 2019).

The role of rigor and determining the appropriate level of rigor are major themes that cut across many conference sessions and conversations among colleagues in our field. In a plenary session at a recent consortium, Neil Morgan, Leigh McAlister, and I proposed a "manifesto" for research that emphasizes asking important questions that are of substantive importance to marketing scholarship and practice while suggesting steps to insure that data availability or method preferences do not drive marketing's research agenda and scholarly output. As others have noted, methodological rigor is vital, but if taken to the extreme, it can delay the timely dissemination of

Mark B. Houston

m.b.houston@tcu.edu

1 Neeley School of Business, Texas Christian University, 2900 Lubbock Avenue, Fort Worth, TX 76129, USA important ideas or, in worst-case scenarios, completely stifle "ambitious papers" (Rust 2018, p. 534).

Rigor is not just a buzzword - it is the basis for having confidence in research findings. In the words of MiriamWebster's Dictionary, it is "the quality of being extremely thorough, exhaustive, or accurate"; the English Language Learner's Dictionary defines it as "the quality or state of being very exact, careful, or strict." Applied to research, these definitions imply that rigor is more than the application of sophisticated and complicated quantitative methodologies. Though much of the debate today is focused on such a narrow conceptualization of rigor, in this editorial, I will argue that we need to rethink and broaden our view of rigor to insure thoroughness, exhaustiveness, accuracy, and care in all phases of the research process. What I will call the "four facets of rigor" range from rigor in developing research questions, to conceptual rigor, methodological rigor, and rigor in crafting a manuscript.

\section{Why do we need to revisit rigor?}

First, let me set the stage for why I believe a broadened view of rigor is critical by looking at the current state of our field. Recently, after being invited to give a "State of Marketing Academia" talk at a doctoral consortium, I reached out via email to 21 people I respected across our discipline, asking them two questions: "What things about our discipline make you hopeful about the future of marketing?" and "What things worry or concern you about the future of our discipline?" My sample was not scientific, but I think it is informative because it included early assistant professors and established veterans, women and men, public and private schools, universities from the U.S., Europe, and Asia, and it spanned the range of topical and method expertise across marketing.

Seven themes emerged from the "hopeful" question and 10 from the "concerns" question (see Table 1). I would note that the number of words (and the emotional intensity) used to express the concerns far exceeded those for the hopeful themes. 
Table 1 Themes from scholars' comments regarding the future of marketing academia

What makes you hopeful? What concerns you?

- We can address BIG questions (for society \& marketing) • Publishing is too often seen as a game (with intense number pressure)

- Talented and creative people comprise our field

- Our field comprises silos (both topical and methodological)

- We nurture our $\mathrm{PhD}$ students and young faculty

- Rigor and methods have become more important than relevance and theory

- Diversity of ideas and people

- Exciting new data \& methods (and multi-method)

- We are increasingly focused on small problems

- $\mathrm{PhD}$ students focus on methods to the exclusion of marketing substance/theory

- Bad behavior (practice \& research integrity)

- Ever-evolving topical domain

- Method specialists versus well-rounded scholars

- With big data, who needs theory?

- Scholarship is devalued in the eyes of the public (and by business school financial models)

- Marketing is ceding important topics to other disciplines (supply chain, IS,

management/ entrepreneurship)

Comparing the "hopeful" themes and the "concern" themes, several real dilemmas clearly exist, and I believe that a narrowed view of rigor has contributed to them. Although scholars believe that marketing is uniquely positioned to address "big" questions (for society and for marketing), we too often end up focused on small problems that can be more confidently addressed with certain kinds of data and specific analysis techniques. Because we increasingly define rigor as methodological sophistication, the nature of $\mathrm{PhD}$ programs in marketing has changed. In the past, $\mathrm{PhD}$ programs sought to develop scholars who had broad-ranging marketing knowledge, with deep training in marketing theory and substance, in addition to methods. PhD training now tends to focus on developing strong methodologists within focused silos, so that these emerging scholars have the toolkits needed to survive the methods gauntlet of top-tier review processes. And, as methodological sophistication is increasingly a focus of these review processes, authors must devote more of their effort and manuscript space to methods instead of theory or the quality of the research question. Several of my respondents articulated concerns that our desire to be respected by scholars in basic disciplines (e.g., economics, psychology) not only heightens a methods focus, but also tends to restrict marketing scholars' attention to questions that would be of interest to a basic discipline.

None of these dilemmas result from evil, ill-intentioned people, but of natural forces that emerge around a discipline that is growing and maturing such that tenure standards and journal standards continue to rise. However, if our field does not make some conscious decisions regarding how they should be resolved (or at least managed), they will continue to intensify, with negative implications for the people in our field and for the quality of our scholarship.

\section{What exactly is rigor?}

Over the past months, I have continued to think about the state of marketing and the role of rigor. I've concluded that, perhaps, we too often argue about rigor without defining exactly what we mean. In doing so, we overlook the fact that rigor is multifaceted. There are several facets of rigor elemental to useful and valid research; methodological rigor is only one facet. ${ }^{1}$ In fact, I'm going to argue that an exclusive focus on methodological rigor actually harms the potential impact of our work. Here are what I see as the four facets of rigor. Note that while methodological rigor gets the most press, I present these facets in what I see as the logical order needed to maximize research quality and impact.

\section{Facet 1: Rigor in designing research questions}

Without an important question, method rigor is of little value. This first facet of rigor involves juxtaposing a rich and systematic understanding of the extant literature with a thorough understanding of real-world phenomena in order to pose important research questions. JAMS, like several other highquality journals in our field, values papers that contribute to the scholarly literature as well as to consumers, managers, and/or policy makers outside of academia. A paper with a cool context, but that ignores a rich body of findings in extant literature, is not optimal; neither is a paper that stems solely from "gaps in the literature," without demonstrating why addressing those gaps matters to anyone (beyond the author).

I challenge authors to build their skill sets in this area, both by staying regularly engaged in the literature and by diving deeply into the substantive context of their chosen research topics. Starting with data and then searching for questions can result in a powerful paper. However, if an entire community of scholars selects research topics based on ease of access to data, it hinders the likelihood that the

\footnotetext{
${ }^{1}$ These ideas are not solely mine. They are a product of interactions over the years with a variety of colleagues and mentors (including Shane Johnson, Neil Morgan, Rob Palmatier, Len Berry, Lisa Scheer, Hari Sridhar, Thorsten Hennig-Thurau, Don Lehmann, John Hulland, Leigh McAlister, Lance Bettencourt, Chris Moorman, Bob Leone, Mike Hutt, and many, many others). Further, these ideas are shaped by insights from numerous sessions at conferences and consortia and from extensive reading on rigor, validity, and philosophies of science, in general.
} 
community will make systematic progress in developing a comprehensive understanding a phenomenon. By having in mind important questions that emerge from a rigorous integration of insights from the literature and from the real world, scholars can be more attuned to how to use data they already possess or can search for the "right" data that allow them to investigate those questions.

\section{Facet 2: Conceptual rigor}

Second, without conceptual rigor, an empirical study is easily misguided; careful, theory-driven thinking is essential for useful research. Bringing theory to bear on a research question and context increases the likelihood that a researcher precisely defines constructs of interest, includes the right set of variables in empirical tests, tests the right set of relationships among variables, and chooses proxies or measures that truly tap the underlying constructs that were intended. Further, stating theory explicitly exposes the researcher's assumptions to the light of day and allows the researcher (and others) to evaluate conceptual logic. Theory does not have to be fully fleshed out before a scholar interacts with their data; in fact, exploratory analyses can often open the scholar's eyes to the true nature of a phenomenon. However, if theory is never developed fully, particularly if it is an afterthought that a scholar addresses only half-heartedly in order to "complete" the manuscript, the potential impact of the work is put at risk.

\section{Facet 3: Methodological and analytical rigor}

This third facet of rigor begins with the care with which data is gathered; useful and valid data require careful and systematic sampling choices, precise execution of data collection procedures, and reliable and justifiable procedures for cleaning/preparing data for analysis. It then continues with analyses that rule out, to an appropriate degree, alternative explanations for findings. The selection and use of any analysis technique requires the scholar to make assumptions, and those assumptions should be clearly stated and compellingly justified. Without methodological and analytical rigor, findings may be due to sampling or selection biases, common source or response biases, endogeneity, and a host of other plausible explanations. This type of rigor is the focus of most current discussion in marketing.

Let me clearly state that regardless of the importance of the underlying research question, without an appropriate degree of method/analysis rigor, a paper's potential impact is reduced. But let's keep in mind that "appropriate" does not have to mean "most sophisticated." Instead, it means doing what is required by the data and the question. Sophistication is not a bad thing, but it can too easily devolve into unneeded complexity (that can hinder communication effectiveness and the accessibility of ideas) or, worse yet, sophistry (that subtly distracts the reader from the primary research question). As Lehmann et al. (2011, p. 162) state, "the more complex approach [must] be worth its complication in terms of producing a different interpretation rather than just, for example, a significant but small improvement in the log-likelihood function."

\section{Facet 4: Rigor in crafting a scholarly manuscript}

Finally, the manuscript is the delivery vehicle for scholarly research. An effective manuscript must compel the reader, present evidence in a convincing manner, proceed in a logical flow, and connect its findings back to prior literature in a way that makes its contribution clear. An important topic that is addressed with useful data but is communicated so poorly that it frustrates readers will struggle to survive the review process. Even if published, weak manuscript-crafting rigor can result in a paper that the field largely ignores as the text obscures its insights rather than highlighting them. My point is not that a manuscript has to be a candidate for a creative writing award; authors simply must ensure that their manuscript is clear and compelling to the reader. Useful advice here is not to treat manuscript rigor as a "last step," but to have it in mind throughout the creation and execution of the research study, contemplating at each step the arguments and evidence that you will need to present in the final manuscript. I also recommend asking trusted peers who are not part of the author team to offer candid, critical feedback on the manuscript's clarity and the degree to which it is compelling and convincing - without such a request, peers often focus primarily on empirical execution.

\section{The bottom line? Balance}

A balance among the four facets of rigor is required. Method rigor without rigor in designing the research question or conceptual rigor may result in a research study that is sophisticated in technique, but not useful to knowledge development or practice. Conversely, great questions and elegant theory with flawed empirical execution will produce findings on which no one can rely. Stated simply, "an important research question" is never a justification for failing to know the methodological standards of the discipline or being sloppy in empirical execution (cf. Lehmann et al. 2011; Moorman et al. 2019). And even with the first three facets of rigor, a lack of manuscriptcrafting rigor can doom a study to low impact.

Although the best studies are strong across all four facets of rigor, it is unlikely that many papers reach the upper limit of each. This is the crux of the debate. How much research- 
question rigor or conceptual rigor are we willing to sacrifice if the method rigor is high enough? I think we would all agree that some papers are important because of the methodological advances they provide. But reviewers in our field are increasingly unwilling to compromise when the imbalance is in the other direction. Specifically, what level of method sophistication are we willing to sacrifice in order to address an important research question or offer initial empirical insights into an important theory idea?

\section{Where to from here?}

There are no clear-cut answers, but I have three strong beliefs that apply. First, tradeoffs across the four facets of rigor are unavoidable and the "ideal" balance will vary across studies - i.e., there is no single, universal ideal. Clearly, every study should not emphasize method rigor above the other facets.

Second, judgment is required to determine the appropriate balance for a given article, starting with the importance of the research question - i.e., there is no easily applied algorithm to optimize the balance. As we seek appropriate levels of rigor, balanced across its four facets, the model is clearly not entirely compensatory. Neither lukewarm papers that are barely at the threshold of each facet of rigor, nor papers that are completely inadequate in any single dimension, are likely to be published or to make an impact. If a question is important enough, then reviewers should be willing to live with, for example, true data limitations that prohibit the use of a more-sophisticated technique. However, the analysis must be executed as precisely as possible and not be fundamentally "wrong" (Lehmann et al. 2011, p. 161). If the method advance is important enough, reviewers should not recommend rejection simply because the substantive question or contribution to theory is modest. However, the authors should discuss compellingly how the method contribution enables scholars to probe specific unanswered questions or to test potential enhancements to theory.

Third, our field, which is an applied discipline, is probably best served by publishing a portfolio of articles that balance the facets of rigor in differing ways. I articulate this third belief because I feel that our field has gotten a bit out of balance, exalting method rigor (narrowly defined, at that) above all others. Other applied fields (such as Management) have found ways to emphasize theory, even as their methodological approaches have advanced. As a science, we need method rigor. However, the topics that span the domain of marketing range from mature to emerging and differ greatly in nature, requiring substantial theoretical advances to understand them. We harm our potential impact if we hold every article to one narrow and rigid view of rigor (i.e., rigor equals quantitative sophistication) to the detriment of addressing the range of big questions we have the potential to answer.

In sum, I believe that if we, as a community of scholars (authors, mentors, reviewers, editors), adopt a broader view of rigor in our work and in our stewardship of the discipline of marketing, we can navigate the dilemmas I noted earlier and make systematic progress in addressing the big issues of our time. At JAMS, we will do our best to use this broadened view of rigor to guide decisions and, in turn, publish a portfolio of papers that exemplify this balance.

\section{References}

Houston, M. B., \& Walker, B. A. (1996). Self-relevance and purchase goals: Mapping a consumer decision. Journal of the Academy of Marketing Science, 24(3), 232-245.

Lehmann, D. R., McAlister, L., \& Staelin, R. (2011). Sophistication in research in marketing. Journal of Marketing, 75(4), 155-165.

Moorman, C., van Heerde, H. J., Page Moreau, C., \& Palmatier, R. W. (2019). JM as a marketplace of ideas. Journal of Marketing, $83(1), 1-7$.

Rust, R. T. (2018). Editorial: Reflections on the review process. International Journal of Research in Marketing, 35(4), 533-535.

Publisher's note Springer Nature remains neutral with regard to jurisdictional claims in published maps and institutional affiliations. 